\title{
Strong disorder for a certain class of directed polymers in a random environment
}

\author{
Philippe CARmonA, Francesco GuERrA ${ }^{\dagger}$ Yueyun Hu ${ }^{\ddagger}$ and Olivier MeJAnE ${ }^{\S}$
}

September 19, 2018

\begin{abstract}
We study a model of directed polymers in a random environment with a positive recurrent Markov chain, taking values in a countable space $\Sigma$. The random environment is a family $(g(i, x), i \geq 1, x \in \Sigma)$ of independent and identically distributed real-valued variables. The asymptotic behaviour of the normalized partition function is characterized: when the common law of the $g(.,$.$) is infinitely divisible and the Markov chain is exponentially recurrent$ we prove that the normalized partition function converges exponentially fast towards zero at all temperatures.
\end{abstract}

\section{Introduction}

In the model of directed polymers in random environment, we study a random Gibbs measure defined on the set of paths (of given length $n$ ) of a stochastic process. Usually one choose for the underlying process a simple random walk on $\mathbb{Z}^{d}$ (see for instance [7],[11] or [3]) or $\mathbb{R}^{d}$ (see [9]). In this paper:

- The stochastic process is an irreducible Markov chain $\left(S_{n}\right)_{n \in \mathbb{N}}$ with countable state space $\Sigma$, defined on a probability space $\left(\Omega, \mathcal{F}, \mathbb{P}_{x}, x \in \Sigma\right)$ with $\mathbb{P}_{x}\left(S_{0}=x\right)=1$.

*P.Carmona: Laboratoire Jean Leray, UMR 6629, Université de Nantes, 92208, F-44322, Nantes cedex 03, e-mail: philippe.carmona@math.univ-nantes.fr

†F.Guerra: Dipartimento di Fisica, Università di Roma "La Sapienza", Instituto Nazionale di Fisica Nucleare, Sezione di Roma 1, Piazzale Aldo Moro, 2 I-00185 Roma, Italy, e-mail: francesco.guerra@roma1.infn.it

†Y.Hu: Laboratoire de Probabilités et Modèles Aléatoires (CNRS UMR-7599), Université Paris VI, 4 Place Jussieu, F-75252 Paris cedex 05, e-mail: hu@proba.jussieu.fr

${ }^{\S}$ O.Mejane: Laboratoire de Statistique et Probabilités, Université Paul Sabatier, 118 route de Narbonne F-31062 Toulouse cedex 04, France, e-mail:Olivier.Mejane@1sp.ups-tlse.fr 
- The environment is a family $(g(i, x), i \geq 1, x \in \Sigma)$ of non-degenerate i.i.d. random variables, distributed as a fixed random variable $g$, defined on a probability space $\left(\Omega^{(g)}, \mathcal{F}^{(g)}, \mathbf{P}\right)$, having some exponential moments

$$
\exists \beta_{0} \in(0,+\infty], \forall|\beta|<\beta_{0}: \quad \mathbf{E}\left[e^{\beta g}\right]=e^{\lambda(\beta)}<+\infty .
$$

- The random energy is the Hamiltonian, defined on the space $\Omega_{n}$ of paths of length $n$ by

$$
H_{n}(g, \gamma)=\sum_{i=1}^{n} g\left(i, S_{i}\right)
$$

(If $\Pi(\cdot, \cdot)$ is the transition matrix of the chain $S$ then

$$
\left.\Omega_{n}=\{\gamma=(\gamma(1), \ldots, \gamma(n)): \Pi(\gamma(i-1), \gamma(i))>0,2 \leq i \leq n\}\right) .
$$

- For a given inverse temperature $\beta>0$, we introduce the Gibbs measure $\langle.\rangle^{(n)}$ on $\Omega_{n}$ and the normalized partition function $Z_{n}(\beta)$ according to the definitions:

$$
\begin{aligned}
& \langle f\rangle \text { (n) } \stackrel{\text { def }}{=} \frac{\mathbb{E}_{x_{0}}\left(f(S) e^{\beta H_{n}(g, S)-n \lambda(\beta)}\right)}{Z_{n}(\beta)}, \\
& Z_{n}(\beta) \stackrel{\text { def }}{=} \mathbb{E}_{x_{0}}\left(e^{\beta H_{n}(S)-n \lambda(\beta)}\right),
\end{aligned}
$$

for any bounded function $f$ from $\Omega_{n}$ to $\mathbb{R}$. We will denote by $\langle\cdot\rangle_{2}^{(n)}$ the product probability measure $\langle.\rangle^{(n)} \otimes\langle.\rangle^{(n)}$ on $\Omega_{n}^{2}$.

It is elementary to check that $\left(Z_{n}(\beta)\right)_{n \geq 0}$ is a $\left(\left(G_{n}\right)_{n \geq 0}, P\right)$ positive martingale, if $\left(\mathcal{G}_{n}\right)_{n \geq 0}$ denotes the natural filtration: $\mathcal{G}_{n}=\sigma(g(k, x), 1 \leq k \leq n, x \in \Sigma)$ for $n \geq 1$ and $\mathcal{G}_{0}=\left\{\emptyset, \Omega^{g}\right\}$. Hence $Z_{n}(\beta) \underset{n \rightarrow \infty}{\longrightarrow} Z_{\infty}(\beta) \geq 0$ almost surely.

Using the terminology of Comets and Yoshida [4], we say there is weak disorder if $Z_{\infty}(\beta)>0$ a.s., and strong disorder if a.s. $Z_{\infty}(\beta)=0$.

When $\left(S_{n}\right)$ is the simple random walk on $\mathbb{Z}^{d}$ and when the environment $g$ is Gaussian, the picture is the following :

- if $d \geq 3$ and $\beta>\beta_{1}$ for some $\beta_{1}>0$, there is strong disorder and almost surely $Z_{n}(\beta)$ converges to zero exponentially fast.

- if $d \geq 3$ and $\beta<\beta_{2}$ for some $\beta_{2}>0$, then there is weak disorder.

- if $d=1,2$ then for any $\beta>0$ there is strong disorder (see [2, 3]) with exponential convergence of $Z_{n}(\beta)$ to 0 if $\beta$ is large enough, but the rate of convergence is still unknown for small $\beta$. 
It is not difficult to prove, by the method of second moment, that there is weak disorder for a "transient" Markov chain when $\beta$ is small, here by "transient" we mean that $\sum_{n, x} \mathbb{P}_{x_{0}}\left(S_{n}=x\right)^{2}<+\infty$.

The aim of this paper is to prove that for a large class of positive recurrent Markov chain, and for fairly general random environments, almost surely $Z_{n}(\beta)$ converges to zero exponentially fast.

$>$ From now on, we shall assume that the Markov chain $\left(S_{n}\right)_{n \in \mathbb{N}}$ is positive recurrent, and that the first return time to $x_{0}, \tau_{x_{0}}=\inf \left\{n \geq 1: S_{n}=x_{0}\right\}$, has small exponential moments

$$
\exists x_{0} \in \Sigma, \exists \kappa>0, \quad \mathbb{E}_{x_{0}}\left[e^{\kappa \tau_{x_{0}}}\right]<\infty
$$

Define

$$
p_{n}(\beta)=\frac{1}{n} \mathbf{E}\left[\log \left(Z_{n}(\beta)\right)\right], \quad 0 \leq \beta<\beta_{0}, \quad \beta_{0} \in(0,+\infty] .
$$

Our main result is the following theorem:

Theorem 1. If the Markov chain $\left(S_{n}\right)_{n \geq 0}$ is irreducible, positive recurrent and satisfies $(\mathbb{E M})$ and if the law of the random environment is infinitely divisible and satisfies (1), then

(a) for small $\beta>0$, the free energy $p(\beta)=\lim _{n \rightarrow \infty} p_{n}(\beta)$ exists and

$$
\frac{1}{n} \log Z_{n}(\beta) \rightarrow p(\beta), \quad \text { a.s. and in } L^{1} .
$$

(b) the function $\beta \in\left[0, \beta_{0}\right) \rightarrow p_{n}(\beta)$ is non increasing.

(c) for all $0<\beta<\beta_{0}$,

$$
\limsup _{n \rightarrow \infty} p_{n}(\beta)<0, \quad \forall \beta \in\left(0, \beta_{0}\right) .
$$

In particular, for any $0<\beta<\beta_{0}$, almost surely $Z_{n}(\beta)$ converges to zero exponentially fast.

This paper is inspired by the works of Francesco Guerra and Fabio Toninelli (see [6]), who developed an interpolation technique to study the high temperature behaviour of the Sherrington-Kirkpatrick mean field spin glass model. The principal ingredient of the proof on the exponential decay is the interpolation between the random Hamiltonian $H_{n}(g, \gamma)$ and a deterministic Hamiltonian.

The paper is organized as follows:

- In Section 2, we evaluate the exponential moments of some additive funstionals whose first consequence is the existence of the free energy $p(\beta)$ for small $\beta>0$. Concentration of measure implies then the a.s. convergence $\frac{1}{n} \log Z_{n}(\beta) \rightarrow p(\beta)$. 
- We devote Section 3 to an integration by parts formula, a feature of infinitely divisible distributions, which entails the monotonicity of free energy (b).

- The last section contains the proof of Theorem 1.

Unless stated otherwise, we assume in the sequel that $\beta \in\left[0, \beta_{0}\right)$ and the random environment $g$ is centered.

\section{Exponential moments}

Recall that $\left(S_{n}\right)$ is a Markov chain taking values in a countable set $\Sigma$ satisfying (EM), and the environment variables $(g(i, x))$ are centered and have some exponential moments (see (10).

Let us omit the dependence on $x_{0}$ of $\tau$ and denote the successive return times to $x_{0}$ by $\tau_{0}=0<\tau_{1}<\tau_{2}<\ldots<\tau_{n}<\ldots$ For a bounded function $f: \Sigma \rightarrow \mathbb{R}$, we define $\bar{f}=\sup _{x \in \Sigma} f(x), \underline{f}=\inf _{x \in \Sigma} f(x)$ and $\|f\|_{\infty}=\sup _{x \in \Sigma}|f(x)|$. The main result of this section is the following theorem:

Theorem 2. Let $f: \Sigma \rightarrow \mathbb{R}$ be a bounded measurable function and $|\beta|<\beta_{0}$ is sufficiently small such that $\lambda(\beta)+2\|f\|_{\infty}<\kappa$.

(i) There exists a unique real number $c(\beta, f) \in[\underline{f}, \bar{f}+\lambda(\beta)]$ such that

$$
\frac{1}{n} \log \mathbb{E}_{x_{0}} \exp \left(\beta \sum_{i=1}^{\tau_{n}} g\left(i, S_{i}\right)+\sum_{i=1}^{\tau_{n}} f\left(S_{i}\right)-c(\beta, f) \tau_{n}\right) \rightarrow 0, \quad \text { a.s. and in } L^{1} .
$$

(ii) We have

$$
\lim _{n \rightarrow \infty} \frac{1}{n} \log \mathbb{E}_{x_{0}} e^{\beta \sum_{i=1}^{n} g\left(i, S_{i}\right)+\sum_{i=1}^{n} f\left(S_{i}\right)}=c(\beta, f), \quad \text { a.s. and in } L^{1} .
$$

The constant $c(\beta, f)$ does not depend on the starting point $x_{0}$, see the forthcoming Remark 9 Taking $\beta=0$ in Theorem 2 , we can evaluate the following Varadhan's type integral

Proposition 3. For any bounded function $f: \Sigma \rightarrow \mathbb{R}$ such that $\|f\|_{\infty}<\kappa / 2$, we have

$$
\lim _{n \rightarrow \infty} \frac{1}{n} \log \mathbb{E}_{x_{0}} e^{\sum_{i=0}^{n-1} f\left(S_{i}\right)}=c(f),
$$

where $c(f) \in[\underline{f}, \bar{f}]$ is the unique real number such that

$$
\mathbb{E}_{x_{0}} \exp \left(\sum_{i=0}^{\tau\left(x_{0}\right)-1} f\left(S_{i}\right)-c(f) \tau\left(x_{0}\right)\right)=1
$$


According to the theory of large deviations, Proposition 3 is well-known at least for the case when $\left(S_{n}\right)$ is a Markov chain with finite states, for example by combining Dembo and Zeitouni ([5], pp. 75) and Ney and Nummelin ([8], Lemma 4.1). See also de Acosta and Ney ([1]) and the references therein for the large deviation principles for a Markov chain.

Taking $f=0$ in Theorem 2 we obtain the existence of the free energy at high temperature (recalling that $g$ is centered):

Proposition 4. Let $|\beta|<\beta_{0}$ be sufficiently small such that $\lambda(\beta)<\kappa$.

(i) There exists a unique real number $c(\beta) \in[0, \lambda(\beta)]$ such that

$$
\frac{1}{n} \log \mathbb{E}_{x_{0}} \exp \left(\beta \sum_{i=1}^{\tau_{n}} g\left(i, S_{i}\right)-c(\beta) \tau_{n}\right) \rightarrow 0, \quad \text { a.s. and in } L^{1}
$$

(ii) We have

$$
\lim _{n \rightarrow \infty} \frac{1}{n} \log \mathbb{E}_{x_{0}} e^{\beta \sum_{i=1}^{n} g\left(i, S_{i}\right)}=c(\beta), \quad \text { a.s. and in } L^{1} .
$$

Before entering into the proof of Theorem 2, we establish a preliminary result on the concentration of measure, which is essentially adapted from Comets, Shiga and Yoshida ([3], Proposition 2.9).

Lemma 5. (Concentration of measure) Let $f: \Sigma \rightarrow \mathbb{R}$ be a bounded measurable function and $|\beta|<\beta_{0}$. Denote by $D_{n}(\beta, f)=\beta \sum_{i=1}^{n} g\left(i, S_{i}\right)+\sum_{i=1}^{n} f\left(S_{i}\right)$ and $\bar{f}=$ $\sup _{x \in \Sigma} f(x)$.

(i) Assume that $\bar{f}+\lambda(\beta)<\kappa$. For any $\varepsilon>0$, there exists a $n_{1}=n_{1}(\beta, f, \varepsilon)<\infty$ such that for all $n \geq n_{1}$,

$$
\mathbf{P}\left(\left|\frac{1}{n} \log \mathbb{E}_{x_{0}} e^{D_{\tau_{n}}}-\frac{1}{n} \mathbf{E} \log \mathbb{E}_{x_{0}} e^{D_{\tau_{n}}}\right|>\varepsilon\right) \leq e^{-\varepsilon^{2 / 3} n^{2 / 3} / 6} .
$$

(ii) For any $\varepsilon>0$, there exists a $n_{2}=n_{2}(\beta, f, \varepsilon)<\infty$ such that for all $n \geq n_{2}$,

$$
\mathbf{P}\left(\left|\frac{1}{n} \log \mathbb{E}_{x_{0}} e^{D_{n}}-\frac{1}{n} \mathbf{E} \log \mathbb{E}_{x_{0}} e^{D_{n}}\right|>\varepsilon\right) \leq e^{-\varepsilon^{2 / 3} n^{2 / 3} / 4} .
$$

(iii) Assume that $\bar{f}+\lambda(\beta)<\kappa$ and fix $1 \leq a \leq b<\infty$. Then for any $\varepsilon>0$, there exists $a n_{3}=n_{3}(a, b, \beta, f, \varepsilon)<\infty$ such that for all $n \geq n_{3}$,

$\mathbf{P}\left(\left|\frac{1}{n} \log \mathbb{E}_{x_{0}}\left(e^{D_{\tau_{n}}} \mid a n \leq \tau_{n} \leq b n\right)-\frac{1}{n} \mathbf{E} \log \mathbb{E}_{x_{0}}\left(e^{D_{\tau_{n}}} \mid a n \leq \tau_{n} \leq b n\right)\right|>\varepsilon\right) \leq e^{-\varepsilon^{2 / 3} n^{2 / 3} / 5}$

with convention $\mathbb{E}_{x_{0}}(\cdot \mid \emptyset) \equiv 1$. 
Proof of Lemma 5, Using the same arguments (martingale decomposition, large deviations for martingale) as that of Comets, Shiga and Yoshida [3] pp. 720721, we obtain (ii) and the following inequality: For any $\varepsilon>0$ and $b>1$, there exists a $n_{4}=n_{4}(b, \beta, f, \varepsilon)<\infty$ such that for all $n \geq n_{4}$ with $\mathbb{P}_{x_{0}}\left(\tau_{n}=k(n)\right)>0$ and $k(n) \leq b n$,

$$
\mathbf{P}\left(\left|\frac{1}{n} \log \mathbb{E}_{x_{0}}\left(e^{D_{\tau_{n}}} 1_{\left(\tau_{n}=k(n)\right)}\right)-\frac{1}{n} \mathbf{E} \log \mathbb{E}_{x_{0}}\left(e^{D_{\tau_{n}}} 1_{\left(\tau_{n}=k(n)\right)}\right)\right|>\varepsilon\right) \leq e^{-\varepsilon^{2 / 3} n^{2 / 3} / 4} .
$$

Observe that

$$
0 \leq \log \mathbb{E}_{x_{0}}\left(e^{D_{\tau_{n}}} 1_{\left(a n \leq \tau_{n} \leq b n\right)}\right)-\max _{a n \leq k \leq b n} \log \mathbb{E}_{x_{0}}\left(e^{D_{\tau_{n}}} 1_{\left(\tau_{n}=k\right)}\right) \leq \log b+\log n,
$$

and for any $u>0$,

$$
\begin{aligned}
& \left\{\left|\max _{a n \leq k \leq b n} \log \mathbb{E}_{x_{0}}\left(e^{D_{\tau_{n}}} 1_{\left(\tau_{n}=k\right)}\right)-\max _{a n \leq k \leq b n} \mathbf{E} \log \mathbb{E}_{x_{0}}\left(e^{D_{\tau_{n}}} 1_{\left(\tau_{n}=k\right)}\right)\right|>u\right\} \\
& \subset \bigcup_{a n \leq k \leq b n}\left\{\left|\log \mathbb{E}_{x_{0}}\left(e^{D_{\tau_{n}}} 1_{\left(\tau_{n}=k\right)}\right)-\mathbf{E} \log \mathbb{E}_{x_{0}}\left(e^{D_{\tau_{n}}} 1_{\left(\tau_{n}=k\right)}\right)\right|>u\right\}
\end{aligned}
$$

The above two observations together with (2) imply (iii). To prove (i), we remark that

$$
\lim _{b \rightarrow \infty} \limsup _{j \rightarrow \infty} \frac{1}{j} \log \mathbf{E}\left(\mathbb{E}_{x_{0}}\left(e^{D_{\tau_{j}}} 1_{\left(\tau_{j} \geq b j\right)}\right)\right)=-\infty .
$$

In fact, we have from Fubini's theorem and Chebychev's inequality that

$$
\begin{aligned}
\mathbf{E}\left(\mathbb{E}_{x_{0}}\left(e^{D_{\tau_{j}}} 1_{\left(\tau_{j} \geq b j\right)}\right)\right) & \leq \mathbb{E}_{x_{0}}\left(e^{(\lambda(\beta)+\bar{f}) \tau_{j}} 1_{\left(\tau_{j}>b j\right)}\right) \\
& \leq e^{-\delta_{0} b j}\left[\mathbb{E}_{x_{0}} e^{\left(\lambda(\beta)+\bar{f}+\delta_{0}\right) \tau}\right]^{j},
\end{aligned}
$$

where $\delta_{0}>0$ denotes a small constant such that $\lambda(\beta)+\bar{f}+\delta_{0}<\kappa$. This yields (3). Finally, applying (iii) to $a=1$ (since $\tau_{n} \geq n$ ) and a sufficiently large $b>0$, we obtain (i).

Proof of Theorem 2, (i) Let $c_{+}=\kappa-\lambda(\beta)-\bar{f}>0$. We shall show that the following function $\psi:\left(-\infty, c_{+}\right) \rightarrow \mathbb{R}$ is well-defined: for any $-\infty<c<c_{+}$,

$$
\psi(c) \stackrel{\text { def }}{=} \lim _{n \rightarrow \infty}\left(\text { a.s. and in } L^{1}\right) \frac{1}{n} \log \mathbb{E}_{x_{0}} \exp \left(\beta \sum_{i=1}^{\tau_{n}} g\left(i, S_{i}\right)+\sum_{i=1}^{\tau_{n}} f\left(S_{i}\right)+c \tau_{n}\right) .
$$

To this end, we shall apply the subadditivity theorem. For notational convenience, denote by

$$
D_{n}=D_{n}(g, S)=\beta \sum_{i=1}^{n} g\left(i, S_{i}\right)+\sum_{i=1}^{n} f\left(S_{i}\right)+c n
$$


Using the strong Markov property at $\tau_{n}$, we have

$$
\mathbb{E}_{x_{0}} e^{D_{\tau_{n+m}}}=\sum_{j} \mathbb{E}_{x_{0}}\left(e^{D_{\tau_{n}}} 1_{\left(\tau_{n}=j\right)}\right) \mathbb{E}_{x_{0}} e^{D_{\tau_{m}}\left(\theta_{j} g, S\right)},
$$

where $\theta_{j}$ denotes the shift operator on $g: \theta_{j} g(i, x)=g(i+j, x)$. By concavity,

$$
\begin{aligned}
\log \mathbb{E}_{x_{0}} e^{D_{\tau_{n+m}}} & =\log \mathbb{E}_{x_{0}} e^{D_{\tau_{n}}}+\log \sum_{j} \frac{\mathbb{E}_{x_{0}}\left(e^{D_{\tau_{n}}} 1_{\left(\tau_{n}=j\right)}\right)}{\mathbb{E}_{x_{0}} e^{D_{\tau_{n}}}} \mathbb{E}_{x_{0}} e^{D_{\tau_{m}}\left(\theta_{j} g, S\right)} \\
& \geq \log \mathbb{E}_{x_{0}} e^{D_{\tau_{n}}}+\sum_{j} \frac{\mathbb{E}_{x_{0}}\left(e^{D_{\tau_{n}}} 1_{\left(\tau_{n}=j\right)}\right)}{\mathbb{E}_{x_{0}} e^{D_{\tau_{n}}}} \log \mathbb{E}_{x_{0}} e^{D_{\tau_{m}}\left(\theta_{j} g, S\right)}
\end{aligned}
$$

Hence

$$
\mathbf{E} \log \mathbb{E}_{x_{0}} e^{D_{\tau_{n+m}}} \geq \mathbf{E} \log \mathbb{E}_{x_{0}} e^{D_{\tau_{n}}}+\mathbf{E} \log \mathbb{E}_{x_{0}} e^{D_{\tau_{m}}}
$$

and

$$
\psi(c) \stackrel{\text { def }}{=} \lim _{n \rightarrow \infty} \frac{1}{n} \mathbf{E} \log \mathbb{E}_{x_{0}} e^{D_{\tau_{n}}}=\sup _{n \geq 1} \frac{1}{n} \mathbf{E} \log \mathbb{E}_{x_{0}} e^{D_{\tau_{n}}}
$$

The a.s. convergence follows from Lemma 5 (i) since $\bar{f}+c+\lambda(\beta)<\kappa$.

As limit of convex and nondecreasing functions, $\psi(\cdot)$ is convex and nondecreasing. Moreover, $\psi:\left(-\infty, c_{+}\right) \rightarrow \mathbb{R}$ is strictly increasing since $\tau_{n} \geq n$. By Jensen's inequality,

$$
\psi(c) \leq \log \mathbb{E}_{x_{0}} e^{(\lambda(\beta)+c+\bar{f}) \tau_{1}},
$$

which implies that $\psi(-(\bar{f}+\lambda(\beta))) \leq 0$. Again using Jensen's inequality and the fact that $g$ is centered, we have

$$
\psi(c) \geq \mathbf{E} \log \mathbb{E}_{x_{0}} e^{D_{\tau_{1}}} \geq \mathbf{E} \log \mathbb{E}_{x_{0}} e^{\beta \sum_{1}^{\tau_{1}} g\left(i, S_{i}\right)+(c+\underline{f}) \tau_{1}} \geq(c+\underline{f}) \mathbb{E}_{x_{0}} \tau_{1},
$$

hence $\psi(-\underline{f}) \geq 0$. It follows that there exists a unique real number $c=c(\beta, f) \in$ $[\underline{f}, \bar{f}+\lambda(\beta)]$ such that $\psi(-c)=0$, proving (i).

(ii) Define

$$
D_{n}=D_{n}(g, S)=\beta \sum_{i=1}^{n} g\left(i, S_{i}\right)+\sum_{i=1}^{n} f\left(S_{i}\right)-c(\beta, f) n
$$

Then by (i),

$$
\frac{1}{j} \log \mathbb{E}_{x_{0}} e^{D_{\tau_{j}}} \rightarrow 0, \quad \text { a.s. and in } L^{1} .
$$

We are going to prove that

$$
\lim _{n \rightarrow \infty} \frac{1}{n} \log \mathbb{E}_{x_{0}} e^{D_{n}}=0, \quad \text { a.s. }
$$


It is not difficult to show that the family $\left(\frac{1}{n} \log \mathbb{E}_{x_{0}} e^{D_{n}}, n \geq 1\right)$ is bounded in $L^{2}$, in fact, by Jensen's inequality, $\frac{1}{n} \log \mathbb{E}_{x_{0}} e^{D_{n}} \geq \frac{\beta}{n} \mathbb{E}_{x_{0}} \sum_{1}^{n} g\left(i, S_{i}\right)+\underline{f}-c(\beta, f)$. On the other hand, since the function $x\left(\in \mathbb{R}_{+}\right) \rightarrow \log ^{2}(x+e)$ is concave,

$$
\mathbf{E}\left(\max \left(0, \frac{1}{n} \log \mathbb{E}_{x_{0}} e^{D_{n}}\right)\right)^{2} \leq \frac{1}{n^{2}} \log ^{2}\left(e+\mathbf{E} \log \mathbb{E}_{x_{0}} e^{D_{n}}\right)=O(1) .
$$

Therefore, the family $\left(\frac{1}{n} \log \mathbb{E}_{x_{0}} e^{D_{n}}, n \geq 1\right)$ is uniformly integrable, which in view of (5) implies that $\frac{1}{n} \mathbf{E} \log \mathbb{E}_{x_{0}} e^{D_{n}} \rightarrow 0$. This proves the $L^{1}$ convergence part of (ii). It remains to show (5) whose proof is divided into two parts.

Upper bound of (5): Notice that $\tau_{j} \geq j$; therefore, we have

$$
\begin{aligned}
\mathbb{E}_{x_{0}} e^{D_{n}} & =\sum_{j=0}^{n-1} \mathbb{E}_{x_{0}}\left(e^{D_{n}} 1_{\left(\tau_{j}<n \leq \tau_{j+1}\right)}\right) \\
& =\sum_{j=0}^{n-1} \mathbb{E}_{x_{0}}\left(\left.e^{D_{\tau_{j}}} 1_{\left(\tau_{j}<n\right)} \mathbb{E}_{x_{0}}\left[e^{D_{k}\left(\theta_{n-k} g, S\right)} 1_{(k \leq \tau)}\right]\right|_{k=n-\tau_{j}}\right) \\
& \leq M_{n} \sum_{j=0}^{n-1} \mathbb{E}_{x_{0}}\left(e^{D_{\tau_{j}}}\right),
\end{aligned}
$$

where

$$
M_{n}=\max _{1 \leq k \leq n} \mathbb{E}_{x_{0}}\left[e^{D_{k}\left(\theta_{n-k} g, S\right)} 1_{\left(k \leq \tau_{1}\right)}\right]
$$

Observe that

$$
\begin{aligned}
\mathbf{E} M_{n} & \leq \sum_{k=1}^{n} \mathbf{E}\left(\mathbb{E}_{x_{0}}\left[e^{D_{k}\left(\theta_{n-k} g, S\right)} 1_{\left(k \leq \tau_{1}\right)}\right]\right) \\
& \leq \sum_{k=1}^{n} \mathbb{E}_{x_{0}}\left[e^{(\lambda(\beta)+\bar{f}-c(\beta, f)) k} 1_{\left(k \leq \tau_{1}\right)}\right] \\
& \leq C n,
\end{aligned}
$$

with

$$
C=\mathbb{E}_{x_{0}}\left[e^{(\lambda(\beta)+\bar{f}-c(\beta, f)) \tau_{1}}\right] \leq \mathbb{E}_{x_{0}}\left[e^{\left(\lambda(\beta)+2\|f\|_{\infty}\right) \tau_{1}}\right]<\infty .
$$

By Borel-Cantelli's lemma, almost surely for all large $n$,

$$
M_{n} \leq n^{3} .
$$

This together with the a.s. convergence in (4) imply the upper bound:

$$
\limsup _{n \rightarrow \infty} \frac{1}{n} \log \mathbb{E}_{x_{0}} e^{D_{n}} \leq 0, \quad \text { a.s. }
$$

Lower bound of (5): By means of (3), for sufficiently large $b>0$,

$$
\mathbf{E}\left(\mathbb{E}_{x_{0}}\left(e^{D_{\tau_{j}}} 1_{\left(\tau_{j} \geq b j\right)}\right)\right) \leq e^{-2 j}
$$


which in view of Borel-Cantelli's lemma yields that $\mathbf{P}(d \omega)$ a.s. for all large $n \geq$ $n_{0}(\omega)$,

$$
\mathbb{E}_{x_{0}}\left(e^{D_{\tau_{j}}} 1_{\left(\tau_{j} \geq b j\right)}\right) \leq e^{-j}
$$

Then by (4) and (7), a.s. for all large $j \geq j_{0}(\varepsilon, \omega)$,

$$
\liminf _{j \rightarrow \infty} \frac{1}{j} \log \mathbb{E}_{x_{0}}\left(e^{D_{\tau_{j}}} 1_{\left(\tau_{j}<b j\right)}\right) \geq 0 .
$$

Let $\varepsilon>0$ be small. We divide the interval $[1, b]$ into $K=K(\varepsilon)=[b / \varepsilon]$ intervals $\left[a_{1}, a_{2}\right), \ldots,\left[a_{K-1}, a_{K}\right)$ with $a_{1}=1, a_{K}=b$ and $a_{k+1}-a_{k}=\frac{b-1}{K}<\varepsilon$ for $1 \leq k \leq$ $K-1$. For any $0 \leq k \leq K-1$, we may repeat the similar argument of subadditivity in (i) and apply the concentration of measure (Lemma 5 (iii)). This yields that

$$
\frac{1}{j} \log \mathbb{E}_{x_{0}}\left(e^{D_{\tau_{j}}} 1_{\left(a_{k} j \leq \tau_{j} \leq a_{k+1} j\right)}\right) \rightarrow \gamma_{k} \quad \text { a.s. and in } L^{1},
$$

for some deterministic constant $\gamma_{k} \in[-\infty, 0]\left(\gamma_{k} \leq 0\right.$ because of (4)). Note that $\gamma_{k}=-\infty$ if and only if for all $j \geq 1, \mathbb{P}_{x_{0}}\left(\tau_{j} \in\left[j a_{k}, j a_{k+1}\right]\right)=0$.

We claim that

$$
\max _{0 \leq k<K} \gamma_{k}=0 \text {. }
$$

Otherwise, since $\gamma_{k}<0$ for each $k<K, \mathbb{E}_{x_{0}}\left(e^{D_{\tau_{j}}} 1_{\left(a_{k} j \leq \tau_{j} \leq a_{k+1} j\right)}\right)$ converges to 0 exponentially fast; then $\mathbb{E}_{x_{0}}\left(e^{D_{\tau_{j}}} 1_{\left(\tau_{j}<b j\right)}\right)=\sum_{k=0}^{K-1} \mathbb{E}_{x_{0}}\left(e^{D_{\tau_{j}}} 1_{\left(a_{k} j \leq \tau_{j} \leq a_{k+1} j\right)}\right)$ would also converge to 0 exponentially fast, which is in contradiction with (8). Then we proved (10).

Now, we proceed to show the lower bound. Choose a fixed $k \in[0, K-1]$ such that $\gamma_{k}=0$. Let $j=\left[\frac{n}{a_{k+1}}\right]$. We have

$$
\begin{aligned}
\mathbb{E}_{x_{0}} e^{D_{n}} & \geq \mathbb{E}_{x_{0}}\left(e^{D_{n}} 1_{\left(j a_{k} \leq \tau_{j}<j a_{k+1}\right)}\right) \\
& =\mathbb{E}_{x_{0}}\left(\left.e^{D_{\tau_{j}}} 1_{\left(j a_{k} \leq \tau_{j}<j a_{k+1}\right)} \mathbb{E}_{x_{0}}\left[e^{D_{\ell}\left(\theta_{n-\ell}, S\right)}\right]\right|_{\ell=n-\tau_{j}}\right) \\
& \geq m_{n} \mathbb{E}_{x_{0}}\left(e^{D_{\tau_{j}}} 1_{\left(j a_{k} \leq \tau_{j}<j a_{k+1}\right)}\right),
\end{aligned}
$$

where by our choice of $j$ and $a_{k}, \ell=n-\tau_{j} \leq n-j a_{k} \leq 2 \varepsilon n$ and

$$
m_{n}=\min _{1 \leq \ell \leq 2 \varepsilon n} \mathbb{E}_{x_{0}}\left[e^{D_{\ell}\left(\theta_{n-\ell g}, S\right)}\right] .
$$

By Jensen's inequality,

$$
\mathbb{E}_{x_{0}}\left[e^{D_{\ell}\left(\theta_{n-\ell} g, S\right)}\right] \geq e^{\mathbb{E}_{x_{0}}\left[D_{\ell}\left(\theta_{n-\ell} g, S\right)\right]} .
$$


Since $f(x)-c(\beta, f) \geq \underline{f}-c(\beta, f) \geq-\left(2\|f\|_{\infty}+\lambda(\beta)\right)>-\kappa$, we have

$$
\mathbb{E}_{x_{0}}\left[D_{\ell}\left(\theta_{n-\ell} g, S\right)\right] \geq \beta \sum_{i=1}^{\ell} \sum_{x} g(i+n-\ell, x) q_{i}(x)-\kappa \ell,
$$

where we write $q_{i}(x)=\mathbb{P}_{x_{0}}\left(S_{i}=x\right)$ for notational convenience. Observe that $\sum_{i=1}^{\ell} \sum_{x}\left(q_{i}(x)\right)^{2}=\sum_{1}^{\ell} \mathbb{P}_{x_{0}}\left(S_{i}=\widetilde{S}_{i}\right) \leq \ell \leq 2 \varepsilon n$, where $\widetilde{S}$ denotes an independent copy of $S$. By Chebychev's inequality, for any $v>0$,

$$
\mathbf{P}\left(\sum_{i=1}^{\ell} \sum_{x} g(i+n-\ell, x) q_{i}(x)<-n^{2 / 3}\right) \leq e^{-v n^{2 / 3}} e^{\Sigma_{1}^{\ell} \sum_{x} \lambda\left(-v q_{i}(x)\right)} \leq e^{-\frac{n^{1 / 3}}{2 \lambda^{\prime \prime}(0)}}
$$

where in the last inequality, we choose $v=\frac{n^{-1 / 3}}{\lambda^{\prime \prime}(0)}$ and use the fact that $\lambda(u) \sim \frac{\lambda^{\prime \prime}(0)}{2} u^{2}$ for small $u$. It turns out that

$$
\mathbf{P}\left(\min _{1 \leq \ell \leq 2 \varepsilon n} \sum_{i=1}^{\ell} \sum_{x} g(i+n-\ell, x) q_{i}(x)<-n^{2 / 3}\right) \leq n e^{-\frac{n^{1 / 3}}{2 \lambda^{\prime \prime}(0)}},
$$

whose sum on $n$ converges. Hence $\mathbf{P}$ a.s. for all large $n \geq n_{0}(\omega), \min _{1 \leq \ell \leq 2 \varepsilon n} \sum_{i=1}^{\ell} \sum_{x} g(i+$ $n-\ell, x) q_{i}(x) \geq-n^{2 / 3}$ and therefore

$$
m_{n} \geq e^{-\beta n^{2 / 3}-2 \varepsilon \kappa n} .
$$

Plugging this into (11) and using (9) with $\gamma_{k}=0$ by our choice of $k$, we obtain that a.s.

$$
\liminf _{n \rightarrow \infty} \frac{1}{n} \log \mathbb{E}_{x_{0}} e^{D_{n}} \geq-2 \varepsilon \kappa
$$

for any $\varepsilon>0$. The lower bound of (5) follows by letting $\varepsilon \rightarrow 0$. This together with the upper bound (6) complete the proof of Theorem 2

Remark 6. When $\beta=0$, the value of $a_{k}$ in (11) can be easily determined by a change of probability measure.

We shall need the following corollary:

Lemma 7. Assume (EM). Let $f$ be a bounded function from $\Sigma$ to $\mathbb{R}$. Then for all $|t|<t_{0}=\frac{\kappa}{2\|f\|_{\infty}}$, the limit

$$
c(t)=\lim _{n \rightarrow \infty} \frac{1}{n} \log \mathbb{E}_{x_{0}}\left(e^{t \sum_{i=1}^{n} f\left(S_{i}\right)}\right)
$$

exists. Moreover, $c$ is differentiable at 0 with $c^{\prime}(0)=\sum_{x \in \Sigma} f(x) \mu(x)$, where $\{\mu(x), x \in$ $\Sigma$ \} denotes the invariant probability measure of $S$. 


\section{Proof.}

Indeed, for $|t|<t_{0}$, the limit $c(t)=\lim _{n \rightarrow \infty} \frac{1}{n} \log \mathbb{E}_{x_{0}}\left(e^{t \sum_{i=1}^{n} f\left(S_{i}\right)}\right)$ exists. It is the unique real number $c$ such that $\phi(c, t)=1$ where $\phi$ is the function

$$
\phi(c, t)=\mathbb{E}_{x_{0}} \exp \left(t \sum_{0}^{\tau\left(x_{0}\right)-1} f\left(S_{i}\right)-c \tau\left(x_{0}\right)\right)
$$

Since $\phi$ is continuously differentiable in $\left(-t_{0}, t_{0}\right) \times J$ with $J$ and open interval, with derivatives

$$
\begin{gathered}
\frac{\partial \phi}{\partial c}=-\mathbb{E}_{x_{0}}\left[\tau\left(x_{0}\right) \exp \left(t \sum_{0}^{\tau\left(x_{0}\right)-1} f\left(S_{i}\right)-c(f) \tau\left(x_{0}\right)\right)\right] \\
\frac{\partial \phi}{\partial t}=\mathbb{E}_{x_{0}}\left[\sum_{0}^{\tau\left(x_{0}\right)-1} f\left(S_{i}\right) \exp \left(t \sum_{0}^{\tau\left(x_{0}\right)-1} f\left(S_{i}\right)-c(f) \tau\left(x_{0}\right)\right)\right]
\end{gathered}
$$

the implicit function theorem entails that $c(t)$ is differentiable in a neighborhood of $t=0$ and

$$
c^{\prime}(0)=\frac{\mathbb{E}_{x_{0}}\left[\sum_{0}^{\tau\left(x_{0}\right)-1} f\left(S_{i}\right)\right]}{\mathbb{E}_{x_{0}}\left[\tau\left(x_{0}\right)\right]} .
$$

Since $f$ is bounded, hence $\mu$-integrable, the ergodic theorem implies

$$
c^{\prime}(0)=\frac{\mathbb{E}_{x_{0}}\left[\sum_{i=0}^{\tau-1} f\left(S_{i}\right)\right]}{\mathbb{E}_{x_{0}}[\tau]}=\lim _{n \rightarrow \infty} \frac{1}{n} \mathbb{E}_{x_{0}}\left[\sum_{i=1}^{n} f\left(S_{i}\right)\right]=\langle\mu, f\rangle,
$$

with $\langle\mu, f\rangle=\sum_{x \in \Sigma} f(x) \mu(x)$.

We now prove that the constant $c(f)$ appearing in Proposition 3 does not really depend on the starting point $x_{0}$. Let $\left(S_{n}\right)$ is a Markov chain taking values in a countable set $\Sigma$. For any $x \in \Sigma$ define

$$
\kappa(x)=\sup \left\{\alpha>0: \mathbb{E}_{x}\left[e^{\alpha \tau(x)}\right]<+\infty\right\}, \quad \text { with } \tau(x)=\inf \left\{n \geq 1: S_{n}=x\right\} .
$$

Let $f: \Sigma \rightarrow \mathbb{R}$ be a bounded function. If $\|f\|_{\infty}<\kappa(x)$ then the following limit exists

$$
c(x, f)=\lim \frac{1}{n} \log \mathbb{E}_{x}\left[e^{A_{n}}\right], \quad \text { with } A_{n}=\sum_{i=0}^{n-1} f\left(S_{n}\right) .
$$

Different state points $x, y$ need to communicate to have the same coefficient.

Lemma 8. If $\left(S_{n}\right)$ is irreducible recurrent and $\|f\|_{\infty}<\frac{1}{2} \inf (\kappa(x), \kappa(y))$ then $c(x, f)=$ $c(y, f)$. 
Proof. Since $\left(S_{n}\right)$ is irreducible recurrent, $\mathbb{P}_{x}(\tau(y)<+\infty)=1$ and there exists $p \geq$ 1 such that $\mathbb{P}_{x}(\tau(y)=p)>0$. Thanks to the strong Markov property,

$$
\mathbb{E}_{x}\left[e^{A_{n}}\right] \geq \mathbb{E}_{x}\left[e^{A_{n}} \mathbf{1}_{(\tau(y)=p)}\right]=\mathbb{E}_{x}\left[e^{A_{p}} \mathbf{1}_{(\tau(y)=p)} \mathbb{E}_{y}\left[e^{A_{n-p}}\right]\right]
$$

Let $\varepsilon>0$. There exists $n_{0}$ such that for all $n \geq n_{0}, \frac{1}{n} \mathbb{E}_{y}\left[e^{A_{n}}\right] \geq c(y, f)-\varepsilon$. Therefore, if $n \geq n_{0}+p$, then

$$
\mathbb{E}_{x}\left[e^{A_{n}}\right] \geq e^{n(c(y, f)-\varepsilon)} \mathbb{E}_{x}\left[e^{A_{p}} \mathbf{1}_{(\tau(y)=p)}\right]
$$

and this yields

$$
c(x, f)=\lim \frac{1}{n} \log \mathbb{E}_{x}\left[e^{A_{n}}\right] \geq c(y, f)-\varepsilon .
$$

Letting $\varepsilon \rightarrow 0$ we get $c(x, f) \geq c(y, f)$. Substituting $x$ for $y$, we obtain $c(x, f)=$ $c(y, f)$.

Remark 9. With the same argument we can prove that $c(x, \beta, f)$ is the same for the starting points $x$ ans $y$, as soon as $\mathrm{1}(\beta)+2\|f\|_{\infty}<\inf (\kappa(x), \kappa(y))$.

\section{Integration by parts formula for infinitely divisible laws}

Recall that the random variable $g$ has small exponential moments. We assume now that it is infinitely divisible, and hence we have a Levy Khinchine formula

$$
ł(\beta)=\log \mathbf{E}\left[e^{\beta g}\right]=c \beta+\frac{\sigma^{2}}{2} \beta^{2}+\int \pi(d u)\left(e^{\beta u}-1-\mathbf{1}_{(|u| \leq 1)} \beta u\right) \quad\left(|\beta|<\beta_{0}\right),
$$

where $c \in \mathbb{R}, \sigma \geq 0$ are constants and $\pi$ is a measure on $\mathbb{R} \backslash\{0\}$ satisfying $\int \pi(d u)(1 \wedge$ $\left.u^{2}\right)<+\infty$.

Lemma 10. If $g$ satisfies (12), then for any bounded differentiable $f$ with bounded derivative, one has the following integration by parts formula:

$$
\mathbf{E}[g f(g)]=c \mathbf{E}[f(g)]+\sigma^{2} \mathbf{E}\left[f^{\prime}(g)\right]+\int_{-\infty}^{+\infty} \pi(d u) u\left[\mathbf{E}[f(g+u)]-\mathbf{1}_{(|u| \leq 1)} \mathbf{E}[f(g)]\right]
$$

Proof. As pointed out by Nicolas Privault, this Lemma can be seen as an easy consequence of much more general integration by parts formulas on the Poisson space (see Picard [10]). Let us give a short proof here: it suffices to prove the formula (13) for $f(x)=e^{i \theta x}$, the extension to more general functions following standard arguments. In that case, $\mathbf{E}[g f(g)]=\mathbf{E}\left[g e^{i \theta g}\right]=e^{\lambda(i \theta)} \lambda^{\prime}(i \theta)$. Since $\lambda^{\prime}(i \theta)=c+\sigma^{2} i \theta+\int_{-\infty}^{+\infty} \pi(d u) u\left(e^{i \theta u}-\mathbf{1}_{(|u| \leq 1)}\right)$, we obtain:

$$
e^{\lambda(i \theta)} \lambda^{\prime}(i \theta)=c e^{\lambda(i \theta)}+\sigma^{2} i \theta e^{\lambda(i \theta)}+\mathbf{E}\left[e^{i \theta g}\right] \int_{-\infty}^{+\infty} \pi(d u) u\left(e^{i \theta u}-\mathbf{1}_{(|u| \leq 1)}\right)
$$




$$
=c \mathbf{E}[f(g)]+\sigma^{2} \mathbf{E}\left[f^{\prime}(g)\right]+\int_{-\infty}^{+\infty} \pi(d u) u\left[\mathbf{E}[f(g+u)]-\mathbf{1}_{(|u| \leq 1)} \mathbf{E}[f(g)]\right] .
$$

We shall now link the derivative of the free energy to $\left\langle L_{n}\left(S^{1}, S^{2}\right)\right\rangle_{2}^{(n)}$, where here and in the sequel, $L_{n}\left(S^{1}, S^{2}\right) \stackrel{\text { def }}{=} \sum_{i=1}^{n} 1_{\left(S_{i}^{1}=S_{i}^{2}\right)}$ denotes the global correlation between the two independent configurations $S^{1}$ and $S^{2}$ (under the same polymers measure $\left.\langle\cdot\rangle^{(n)}\right)$. Recall that $I$ is an open interval, chosen as big as possible, such that $0 \in I \subset$ $\{\beta: 1(\beta)<+\infty\}$.

Proposition 11. If $g$ satisfies (12), then there exists $c_{1}>0$, depending on the law of $g$ and on $\beta$, such that $\forall \beta \in I \cap(0, \infty)$

$$
p_{n}^{\prime}(\beta) \leq-\frac{c_{1}}{n} \mathbf{E}\left[\left\langle L_{n}\left(S^{1}, S^{2}\right)\right\rangle_{2}^{(n)}\right] \text {. }
$$

In particular, for all $n \geq 1, \beta \mapsto p_{n}(\beta)$ is non increasing.

Moreover if $2 \beta \in I \cap(0, \infty)$, there exists $c_{2}(\beta)>0$ such that

$$
p_{n}^{\prime}(\beta) \geq-\frac{c_{2}}{n} \mathbf{E}\left[\left\langle L_{n}\left(S^{1}, S^{2}\right)\right\rangle_{2}^{(n)}\right] .
$$

Proof. In the sequel we write $\langle$.$\rangle instead of \langle.\rangle^{(n)}$. The first step is the following identity:

$$
n p_{n}^{\prime}(\beta)=\mathbf{E}\left[\left\langle\sum_{i=1}^{n} g\left(i, S_{i}\right)\right\rangle\right]-n \lambda^{\prime}(\beta)=\sum_{i, x} \mathbf{E}\left[\left[g(i, x) F_{i, x}(g(i, x))\right]\right]-n \lambda^{\prime}(\beta),
$$

where we have set, for each $(i, x)$ :

$$
F_{i, x}(u) \stackrel{\text { def }}{=} \frac{\mathbb{E}\left[\mathbf{1}_{\left(S_{i}=x\right)} \exp \left(\beta \sum_{(j, y) \neq(i, x)} g(j, y) \mathbf{1}_{\left(S_{j}=y\right)}+\beta u \mathbf{1}_{\left(S_{i}=x\right)}\right)\right]}{\mathbb{E}\left[\exp \left(\beta \sum_{(j, y) \neq(i, x)} g(j, y) \mathbf{1}_{\left(S_{j}=y\right)}+\beta u \mathbf{1}_{\left(S_{i}=x\right)}\right)\right]}, \quad u \in \mathbb{R} .
$$

Since $F_{i, x}$ is a random function depending only on $(g(j, y),(j, y) \neq(i, x))$, it is independent of $g(i, x)$, so by Lemma 10 one has for each fixed $(i, x)$ :

$$
\begin{aligned}
\mathbf{E}\left[g(i, x) F_{i, x}(g(i, x))\right] & =c \mathbf{E}\left[F_{i, x}(g(i, x))\right]+\sigma^{2} \mathbf{E}\left[F_{i, x}^{\prime}(g(i, x))\right] \\
& +\int_{-\infty}^{+\infty} \pi(d u) u\left[\mathbf{E}\left[F_{i, x}(g(i, x)+u)\right]-\mathbf{1}_{(|u| \leq 1)} \mathbf{E}\left[F_{i, x}(g(i, x))\right]\right]
\end{aligned}
$$

Here one easily obtains that $F_{i, x}^{\prime}(u)=\beta F_{i, x}(u)\left[1-F_{i, x}(u)\right]$. In particular, one has

$$
F_{i, x}^{\prime}(g(i, x))=\beta\left\langle\mathbf{1}_{\left(S_{i}=x\right)}\right\rangle\left(1-\left\langle\mathbf{1}_{\left(S_{i}=x\right)}\right\rangle\right) .
$$


Moreover,

$$
F_{i, x}(g(i, x)+u)=\frac{\left\langle\mathbf{1}_{\left(S_{i}=x\right)}\right\rangle e^{\beta u}}{\left\langle\mathbf{1}_{\left(S_{i} \neq x\right)}\right\rangle+\left\langle\mathbf{1}_{\left(S_{i}=x\right)}\right\rangle e^{\beta u}},
$$

so that formula (17) leads to:

$$
\begin{aligned}
\mathbf{E}\left[g(i, x) F_{i, x}(g(i, x))\right] & =c \mathbf{E}\left[\left\langle\mathbf{1}_{\left(S_{i}=x\right)}\right\rangle\right]+\sigma^{2} \beta \mathbf{E}\left[\left\langle\mathbf{1}_{\left(S_{i}=x\right)}\right\rangle\left(1-\left\langle\mathbf{1}_{\left(S_{i}=x\right)}\right\rangle\right)\right] \\
& +\int_{-\infty}^{+\infty} \pi(d u) u\left[\mathbf{E}\left[\frac{\left\langle\mathbf{1}_{\left(S_{i}=x\right)}\right\rangle e^{\beta u}}{\left\langle\mathbf{1}_{\left(S_{i} \neq x\right)}\right\rangle+\left\langle\mathbf{1}_{\left(S_{i}=x\right)}\right\rangle e^{\beta u}}\right]-\mathbf{1}_{(|u| \leq 1)} \mathbf{E}\left[\left\langle\mathbf{1}_{\left(S_{i}=x\right)}\right\rangle\right]\right]
\end{aligned}
$$

Then, using that $\lambda^{\prime}(\beta)=c+\sigma^{2} \beta+\int_{-\infty}^{+\infty} \pi(d u) u\left(e^{\beta u}-\mathbf{1}_{(|u|<1)}\right)$ and remembering that $\sum_{i, x}\left\langle\mathbf{1}_{\left(S_{i}=x\right)}\right\rangle=n$, equation 10 becomes

$n p_{n}^{\prime}(\beta)=-\sigma^{2} \beta \mathbf{E}\left[\sum_{i, x}\left\langle\mathbf{1}_{\left(S_{i}=x\right)}\right\rangle^{2}\right]-\sum_{i, x} \int_{-\infty}^{+\infty} \pi(d u) u \mathbf{E}\left[\frac{\left\langle\mathbf{1}_{\left(S_{i}=x\right)}\right\rangle^{2} e^{\beta u}\left(e^{\beta u}-1\right)}{1+\left\langle\mathbf{1}_{\left(S_{i}=x\right)}\right\rangle\left(e^{\beta u}-1\right)}\right]$.

Now we prove that

$$
\inf _{0 \leq a \leq 1} \int_{-\infty}^{+\infty} \pi(d u) u \frac{e^{\beta u}\left(e^{\beta u}-1\right)}{1+a\left(e^{\beta u}-1\right)}>0
$$

as soon as $\pi() \neq$.0 . On the one hand, if $\operatorname{supp}(\pi) \cap \mathbb{R}_{+} \neq \emptyset$, then for all $0 \leq a \leq 1$,

$$
\int_{0}^{+\infty} \pi(d u) u \frac{e^{\beta u}\left(e^{\beta u}-1\right)}{1+a\left(e^{\beta u}-1\right)} \geq \int_{0}^{+\infty} \pi(d u) u\left(e^{\beta u}-1\right)>0 .
$$

On the other hand, if $\operatorname{supp}(\pi) \cap \mathbb{R}_{-} \neq \emptyset$, then for all $0 \leq a \leq 1$,

$$
\int_{-\infty}^{0} \pi(d u)|u| \frac{e^{\beta u}\left(1-e^{\beta u}\right)}{1-a\left(1-e^{\beta u}\right)} \geq \int_{-\infty}^{0} \pi(d u) e^{\beta u}|u|\left(1-e^{\beta u}\right)>0 .
$$

In all cases, 18 is true for all $\pi() \neq$.0 , so there exists $c_{1}>0$ such that

$$
n p_{n}^{\prime}(\beta) \leq-c_{1} \mathbf{E}\left[\sum_{i, x}\left\langle\mathbf{1}_{\left(S_{i}=x\right)}\right\rangle^{2}\right]
$$

$c_{1}$ being positive because $\pi() \neq$.0 or $\sigma>0$, since the law of $X$ is non-degenerate. This leads the upper bound (14) thanks to the following identity:

$$
\sum_{i, x}\left\langle\mathbf{1}_{\left(S_{i}=x\right)}\right\rangle^{2}=\left\langle L_{n}\left(S^{1}, S^{2}\right)\right\rangle_{2}^{(n)} .
$$

The lower bound (15) can be deduced in the same way, using that

$$
\begin{aligned}
\sup _{0 \leq a \leq 1} \int_{-\infty}^{+\infty} \pi(d u) u \frac{e^{\beta u}\left(e^{\beta u}-1\right)}{1+a\left(e^{\beta u}-1\right)} & \leq \int_{0}^{+\infty} \pi(d u) u e^{\beta u}\left(e^{\beta u}-1\right) \\
& +\int_{-\infty}^{0} \pi(d u)|u|\left(1-e^{\beta u}\right) \\
& \stackrel{\text { def }}{=} c^{\prime},
\end{aligned}
$$

because $c^{\prime}<\infty$ provided that $2 \beta \in I$. 


\section{Strong disorder: Proof of Theorem 1}

The part (a) of Theorem 1 follows from Proposition 4, whereas the part (b) from Proposition 11 To show the part (c), we make use of the monotonicity of $\beta \rightarrow$ $p_{n}(\beta)$, then it suffices to prove that $\limsup p_{n}(\beta)<0$ for $\beta>0$ small enough. Recall that $\mathbf{E}(g)=\lambda^{\prime}(0)=0$. Then for all $q=(q(i, x), i \geq 1, x \in \Sigma) \in \mathbb{R}^{\mathbb{N}^{*} \times \Sigma}$, one has, using Jensen's inequality:

$$
\begin{aligned}
p_{n}(\beta) & =\frac{1}{n} \mathbf{E}\left[\log \mathbb{E}_{x_{0}}\left[\left(e^{\beta \sum_{i, x} g(i, x) \mathrm{I}_{S_{i}=x}-n \lambda(\beta)}\right)\right]-\beta \sum_{i, x} g(i, x) q(i, x)\right] \\
& =\frac{1}{n} \mathbf{E}\left[\log \mathbb{E}_{x_{0}}\left[\left(e^{\beta \sum_{i, x} g(i, x)\left(\mathrm{I}_{S_{i}=x}-q(i, x)\right)-n \lambda(\beta)}\right)\right]\right] \\
& \leq \frac{1}{n} \log \mathbb{E}_{x_{0}}\left(e^{\sum_{i, x} \lambda\left(\beta\left[\mathrm{I}_{S_{i}=x}-q(i, x)\right]\right)-n \lambda(\beta)}\right),
\end{aligned}
$$

Let us choose $q(i, x)=\mu(x)$ (the invariant probability measure of $S$ ) for all $(i, x) \in$ $\mathbb{N}^{*} \times \Sigma$ and let us fix $\varepsilon>0$. There exists $\beta_{\varepsilon}>0$ such that $\forall 0<\beta<\beta_{\varepsilon}, \frac{1-\varepsilon}{2} \beta^{2} \lambda^{\prime \prime}(0) \leq$ $\lambda(\beta) \leq \frac{1+\varepsilon}{2} \beta^{2} \lambda^{\prime \prime}(0)$. Thus, for $0<\beta<\beta_{\varepsilon}$,

$$
\begin{aligned}
p_{n}(\beta) & \leq \frac{1}{n} \log \mathbb{E}_{x_{0}}\left(e^{\frac{1+\varepsilon}{2} \beta^{2} \lambda^{\prime \prime}(0) \sum_{i, x}\left(I_{S_{i}=x}-\mu(x)\right)^{2}-n \frac{1-\varepsilon}{2} \beta^{2} \lambda^{\prime \prime}(0)}\right) \\
& \leq \frac{1}{n} \log \mathbb{E}_{x_{0}}\left(e^{-(1+\varepsilon) \beta^{2} \lambda^{\prime \prime}(0) \sum_{i=1}^{n} \mu\left(S_{i}\right)}\right)+\varepsilon \lambda^{\prime \prime}(0) \beta^{2}+\frac{1+\varepsilon}{2} \beta^{2} \lambda^{\prime \prime}(0)\|\mu\|^{2},
\end{aligned}
$$

with $\|\mu\|^{2}=\sum_{x \in \Sigma} \mu^{2}(x)$.

Applying Lemma [7 to $f(x)=-\mu(x)$, one deduces the existence of $J=\left[-t_{0}, t_{0}\right]$ and of $c_{\mu}$ defined on $J$ such that $\forall t \in J, \frac{1}{n} \log \mathbb{E}_{x_{0}}\left(e^{t \sum_{i=1}^{n} \mu\left(S_{i}\right)}\right) \underset{n \rightarrow \infty}{\longrightarrow} c_{\mu}(t)$ and $\forall t \in$ $J, c_{\mu}(t) \leq-(1-\varepsilon) t\|\mu\|^{2}$, since $c_{\mu}^{\prime}(0)=-\|\mu\|^{2}$. Hence for $\beta$ small enough one concludes that $\limsup _{n \rightarrow \infty} p_{n}(\beta) \leq-\frac{1}{2} \beta^{2}\|\mu\|^{2} \lambda^{\prime \prime}(0)$ and thus $\lim _{\sup } \operatorname{sum}_{n \rightarrow \infty} p_{n}(\beta)<0$ since $\lambda^{\prime \prime}(0)=\operatorname{Var}(g)>0$. Finally, for any $0<\beta<\beta_{0}$, we deduce from the property of concentration of measure (Lemma[5] (ii)) that almost surely, $Z_{n}(\beta)$ converges to 0 exponentially fast.

\section{References}

[1] A. de Acosta and P. Ney. Large deviation lower bounds for arbitrary additive functionals of a Markov chain. Ann. Probab. 26 (1998), no. 4, 1660-1682.

[2] Ph. Carmona and Y. Hu. On the partition function of a directed polymer in a Gaussian random environment. Probab. Theory Related Fields, 124(3):431-457, 2002.

[3] F. Comets, T. Shiga, and N. Yoshida. Directed polymers in random environment: path localization and strong disorder. Bernoulli (2003) 9(4):705-723, 2003. 
[4] F. Comets and N. Yoshida. Brownian directed polymers in random environment. preprint, 2003.

[5] A. Dembo and O. Zeitouni. Large deviations techniques and applications. Second edition. Springer-Verlag, New York, 1998.

[6] F. Guerra and F. L. Toninelli. Quadratic replica coupling in the Sherrington-Kirkpatrick mean field spin glass model. J. Math. Phys., 43(7):3704-3716, 2002.

[7] J. Z. Imbrie and T. Spencer. Diffusion of directed polymers in a random environment. J. Statist. Phys., 52(3-4):609-626, 1988.

[8] P. Ney and E. Nummelin. Markov additive processes. I. Eigenvalue properties and limit theorems. Ann. Probab. 15 (1987), no. 2, 561-592.

[9] M. Petermann. Superdiffusivity of directed polymers in a random environment. part of PHD thesis, 2000.

[10] J. Picard. Formules de dualité sur l'espace de Poisson. Ann. Inst. H. Poincaré Probab. Statist., 32(4):509-548, 1996.

[11] Y. Sinai. A remark concerning random walks with random potentials. Fundamenta Mathematicae, 147(2):173-180, 1995. 